\title{
UNA PROPUESTA DE ESTUDIO DE PROCESOS DE INCLUSIÓN Y ACCESO A DERECHOS EN JUVENTUDES Y GÉNERO
}

\author{
UMA PROPOSTA PARA ESTUDAR OS PROCESSOS DE INCLUSÃO E ACESSO A \\ DIREITOS NA JUVENTUDE E NO GÊNERO
}

Yussef Becher ${ }^{1}$

\section{Resumen}

En un contexto en donde la inclusión y el acceso a derechos se han vuelto dos significantes recurrentes en discursos políticos y mediáticos, vale la pena que desde las ciencias sociales propongamos miradas teóricas. Por ello este artículo tiene tal objetivo respecto de los estudios sociales en juventudes y su clivaje con género por cuanto es en esa área en la cual contamos con experiencias de investigación.

Una mirada teórica implica un posicionamiento epistemológico ante un objeto de estudio: en nuestro caso dicha mirada es socio-antropológica. Una perspectiva de tales características debe reparar en dos elementos que confluyen en los procesos de inclusión y acceso a derechos: por una parte la responsabilidad estatal y por otra los efectos subjetivos sobre quienes son sus destinatarios.

Asimismo, el texto propone tres dimensiones de análisis -o variables conceptuales- como propuesta metodológica a partir de la cual pueden explorarse los aspectos teóricos señalados en la primera parte. Por último, se presentan algunos resultados empíricos -a modo de prueba de las variables- enmarcados en investigaciones sobre los temas que se abordan en este texto.

Palabras clave: Propuesta de Estudio; Inclusión; Acceso a Derechos; Juventudes; Género

\section{Resumo}

Em um contexto em que a inclusão e o acesso aos direitos se tornaram dois significantes recorrentes nos discursos políticos e midiáticos, é necessário que, a partir das ciências sociais, proponham-se perspectivas teóricas. Por essa razão, este artigo tem esse objetivo em relação aos estudos sociais na juventude e sua clivagem com o gênero, porque é nessa área em que temos experiências de pesquisa. Uma perspectiva teórica implica um posicionamento epistemológico diante de um objeto de estudo: no nosso caso, essa visão é socioantropológica. Uma perspectiva de tais características deve dar atenção a dois elementos que convergem nos processos de inclusão e acesso a direitos: de um lado, a responsabilidade do Estado e, de outro, os efeitos subjetivos sobre quem são os destinatários. Da mesma forma, o texto propõe três dimensões de análise - ou variáveis conceituais - como uma proposta metodológica a partir da qual os aspectos teóricos indicados na primeira parte podem ser explorados. Por fim, alguns resultados empíricos são apresentados - como teste das variáveis - enquadrados em pesquisas sobre os tópicos abordados neste texto.

Palavras-chave: Proposta de Estudo; Inclusão; Acesso a Direitos; Juventude; Gênero.

\footnotetext{
1 Abogado. Magister en Sociedad e Instituciones (UNSL). Doctorando en Ciencias Sociales (FLACSO Argentina). Becario doctoral del CONICET. Docente investigador de la UNSL. E-mail: yussefbe@hotmail.com
} 


\section{LA INCLUSIÓN SOCIAL Y EL ACCESO A DERECHOS: ENTRE EN EL SENTIDO COMÚN Y EL ANÁLISIS CIENTÍFICO}

En los últimos quince años de la historia contemporánea argentina tanto uno como otro término se han convertido en significantes de uso recurrente por parte del discurso político y mediático. Ya en el siglo XIX se le atribuía al escritor francés Max Jacobs la conocida frase: el sentido común es el menos común de todos los sentidos. Con ello podemos reconocer la influencia de los dispositivos políticos y mediáticos en la construcción de dicho sentido y de allí la oportunidad única de las ciencias sociales: superar ese discurso y contribuir con rigurosidad científica a la construcción de un significante contemporáneo que se ha vuelto de frecuente uso por parte del discurso social.

La búsqueda bibliográfica suele ser uno de los primeros pasos que antecede a la selección o a la construcción de nuestras categorías teóricas que, al mejor estilo de una lupa, serán utilizadas para observar el fenómeno social que nos proponemos estudiar. No es una tarea sencilla la de ubicar la bibliografía apropiada a nuestra temática de interés y de allí el agobio -y en algunos casos angustia- que suele acompañar esa instancia inicial. Dada la multiplicidad de posibilidades que la vida contemporánea ofrece para acceder a material bibliográfico de distinto tipo -y en ello el importante papel que corresponde a las publicaciones digitales que comenzaron a popularizarse en nuestra región a fines de la década de 1990- esa búsqueda suele ser aun más agobiante.

Quien ha tenido que transitar por esos momentos puede comprender la importancia de reconocer un texto en donde claramente se presente una propuesta teórico-metodológica de abordaje de categorías teóricas, pues allí el lector tiene la oportunidad de leerlo o desecharlo de acuerdo con sus intereses. A partir de ello, nuestro texto propone una particular mirada sobre dos categorías que han incrementado su uso en estos últimos tiempos, ya sea de parte de la comunidad académica o de otras. Sin dudas, nuestra intención es incidir en la primera y con ello en las segundas pues hoy ya no pueden escindirse, menos aun cuando se trata de significantes instalados en el discurso social, una de otra.

La inclusión social y el acceso a derechos son de esas herramientas de análisis propias de las ciencias sociales que no escapan a su polisemia y de allí la multiplicidad de abordajes existentes. En nuestro caso y debido a nuestras propias experiencias de investigación en estudios sociales de juventudes y su clivaje con género, hemos optado por una mirada socio- 
antropológica. Dicha perspectiva -tal como afirman Das y Poole (2008)- coloca especial énfasis en la exploración por las prácticas y políticas subjetivas que moldean las prácticas políticas de regulación y disciplinamiento de aquello que llamamos Estado. De modo que esa configuración socio-estatal es el espacio en donde se producen las subjetividades y las regulaciones estatales.

Por otra parte, las desigualdades de género suponen un desafío especial para los procesos inclusivos pues en tal caso no solo se trata de igualar sino de equiparar entre quienes se enfrentan a situaciones de discriminación que son estructurales. En tal sentido, nos parece que la distinción entre igualdad de oportunidades y de posiciones -introducida teóricamente por el sociólogo francés François Dubet (2011)- aporta pistas interesantes para pensar en torno a ello.

Finalmente, en su última parte, el texto ensaya una trilogía de variables conceptuales cuya evaluación queda a cargo de los lectores- que consideramos apropiadas para bucear en las múltiples realidades que ofrecen los diferentes escenarios en donde se construyen experiencias inclusivas y de derechos. Tales variables integran una tesis doctoral en curso en el marco del programa de la Facultad Latinoamericana de Ciencias Sociales (FLACSO) y de un proyecto de investigación de la Universidad Nacional de San Luis en Argentina.

\section{LA PROPUESTA DE ESTUDIO: ESTADO Y SUBJETIVIDADES}

En la primera década del nuevo siglo transcurrida en nuestra región, los propósitos de inclusión y acceso a derechos se objetivaron -en la mayoría de los países- en políticas sociales (Guendel, 2007; Andrenacci, 2010) y esto es consecuente con los objetivos que todo Estado que se denomina democrático debe garantizar puesto que el goce efectivo de los derechos sociales constituye un plafón necesario para el ejercicio de los civiles (Dubet, 2011). Aunque los diferentes contextos sociopolíticos inciden pues tales objetivos no siempre se han cumplido del mismo modo y por ello las políticas sociales son consideradas una construcción social en la cual influyen los diferentes condicionantes culturales y económicos (Biernat y Ramaciotti, 2012).

En los ámbitos que corresponden a las juventudes y a la disminución de desigualdades de género, las acciones estatales llevadas a cabo en Argentina durante ese periodo constituyeron importantes avances en orden a reducir algunas de tales brechas aunque, sin dudas, otras persisten de manera insistente. Tanto en lo que refiere a la juventud como al 
género, la principal brecha se ubica en la esfera ligada al empleo, ya sea por precarización o por no reconocer las tareas de cuidado. ${ }^{2}$

Tal como hemos visto, desde una perspectiva socio-antropológica, debemos reconocer que todo proceso social que involucra al Estado implica identificar su expresa incidencia, como así también de las subjetividades que se construyen a partir de la vinculación con aquel. Por ello nuestra propuesta para el estudio de la inclusión y el acceso a derechos advierte -tal como afirman Burchardt et. al. (2002)- la necesidad de reconocer que se trata de un problema desde el punto de vista del Estado y de los ciudadanos.

\section{El papel del Estado}

A fin de indagar el rol estatal en la configuración de los procesos inclusivos y de acceso a derechos, se puede acudir a diferentes perspectivas teóricas: entre ellas, la de Esping-Andersen (1990) y su teoría sobre los regímenes de bienestar, o bien la de Amartya Sen (1997 y 2000) y las human capabilities o del enfoque de derechos (Abramovich, 2004; 2006; ONU, 2006; Pautassi, 2010), entre muchas otras.

En la contemporaneidad argentina, los estudios sociales sobre intervenciones estatales muestran que esos son los tres enfoques a partir de los cuales suele ser estudiado el rol estatal.

La teoría de los estados de bienestar reconoce sus orígenes en el texto The three worlds of welfare capitalism de Gosta Esping-Andersen (1990). Allí el sociólogo danés señala que los principales componentes para definir el modo en que se instauran los mencionados estados surgen de la presencia de los siguientes elementos: la calidad de los derechos sociales; la estratificación social; y la relación existente entre el Estado, el mercado y la familia. De esa manera define tres formas en las que pueden estructurarse los regímenes del Estado de Bienestar: liberales, conservadores o corporativistas y socialdemócratas. En ellos el papel de las políticas sociales será distinto de acuerdo al régimen de que se trate. En el liberal tienen una función residual pues predominan los seguros sociales y la protección social-que cuentan con

\footnotetext{
2 En los estudios realizados por Salvia y Tuñon $(2006 ; 2007)$ se pueden advertir -desde una perspectiva cuantitativa- las limitaciones de las políticas de empleo juveniles implementadas a inicios del nuevo siglo por cuanto no han logrado cumplir con sus objetivos de capacitación e inserción en el mercado laboral formal. Por su parte, en Núñez et. al. (2015) se describen los efectos cualitativos de la construcción de una clase social precarizada en torno a las juventudes pues el problema no se limita a los últimos años sino que es propio de la estructura sociohistórica de nuestro país. En cuanto a las mujeres, el último informe de la Organización de Naciones Unidas (2010) y los textos de Pautassi (2015) y Rodríguez Enríquez (2015) mencionan la centralidad del trabajo femenino no remunerado de cuidado en las actuales desigualdades sociales de género.
} 
escasos recursos- otorgados a aquellos que comprueben pertenecer al sector de la población que menos ingresos posee. El corporativista o conservador es aquel que intenta conservar las diferencias de clase o estatus y en ello la posibilidad de resguardar derechos prioritariamente para los que pertenecen a tales estratos. En ellos la Iglesia Católica -como institución dominante- tiende a mantener el modelo de familia tradicional y de allí que la seguridad social suele excluir a las mujeres y los subsidios familiares estimulan la maternidad. Debido a la centralidad de la familia -y principalmente de la mujer- como proveedora de bienestar, se considera que el Estado solo debe intervenir cuando aquella pierde capacidad para atender las necesidades de sus miembros. Por último, en un Estado de Bienestar socialdemócrata la prioridad se encuentra en la consolidación de nuevas clases medias. Allí predominan programas universalistas que intentan adaptarse a las expectativas de las diferentes clases sociales (EspingAndersen, 1990).

Los trabajos académicos más recientes en Argentina que analizan acciones estatales desde aquel enfoque corresponden al Grupo de Trabajo Interdisciplinario Derechos Sociales y Políticas Públicas. El mismo realiza sus actividades en el Instituto de Investigaciones Jurídicas y Sociales Ambrosio Gioja de la Facultad de Derecho y de la Facultad de Ciencias Sociales de la Universidad de Buenos Aires.

En la última compilación del equipo de investigación -que analiza distintas políticas sociales implementadas en el último decenio- se señala que las modificaciones en la estructura de riesgos sociales no han tenido correlato en los ámbitos institucionales dispuestos para darles respuesta pues allí no se han realizado las reformas necesarias. Además de ello, la principal brecha de bienestar se ubica en la necesidad de incorporar en los programas sociales la perspectiva género sensitiva (Arcidiácono, 2015; Gamallo, 2015; Pautassi, 2015).

En el enfoque de las human capabilities el principal teórico ha sido el Premio Nobel de Economía Amartya Sen. En esa perspectiva las principales categorías teóricas para definir las políticas sociales giran en torno a los conceptos de desarrollo -y su ligazón con la libertad- y capacidades humanas. Sen (2000) considera que el desarrollo no es simple acumulación de riqueza, crecimiento del Producto Bruto Interno y otras variables relacionadas con la renta, sin embargo, es la utilidad de la riqueza la que delimita las libertades que podemos conseguir. El punto de partida de su enfoque es la identificación de la libertad como objeto principal del desarrollo. A partir de allí, el objetivo de análisis de la política social es la constatación de la conexión empírica entre tales conceptos (Tonon, 2008). 
Asimismo, el autor advierte que el mundo niega libertades básicas a un inmenso número de personas. Ello exige que el desarrollo elimine las principales fuentes de privación de la libertad; la pobreza y la tiranía; la escasez de oportunidades económicas; las privaciones sociales sistemáticas y el abandono en que pueden encontrarse los servicios públicos. Por ello el desarrollo no puede dejar de lado la consideración acerca del estilo de vida que llevan a cabo las personas y sus libertades reales (Sen, 2003).

Sen (1997) afirma que un elemento para superar la desigualdad -mediante la obtención de mayores libertades y sus efectos sobre el desarrollo- reside en la posibilidad de que los servicios sociales contribuyan a la construcción de human capabilities. El economista distingue entre los conceptos de capital humano y capacidad humana. El primero de ellos se vincula con la capacidad del ser humano para incrementar sus posibilidades de producción, por el contrario, capacidad humana se refiere a la viabilidad de adquirir posibilidades reales de elección. Al mismo tiempo, una concepción restringida de capital humano puede incorporarse en una perspectiva amplia de capacidad humana, de allí la mayor obtención de libertades y desarrollo.

Las investigaciones más recientes que vinculan programaciones sociales y human capabilities corresponden a la Human Development and Capability Association y la International Society for Quality -of- Life Studies (ISQOLS). Ambas tienen representantes en Argentina.

La última perspectiva teórica que nos parece relevante mencionar en esta revisión, por cuanto ha adquirido importante relevancia en los estudios sobre intervenciones estatales, es la de derechos. El Estado al ratificar tratados internacionales con otros países -de acuerdo con las pautas de los organismos internacionales- adquiere una serie de compromisos que debe cumplir al llevar a cabo sus acciones. Las últimas pueden canalizarse por medio de políticas sociales. Allí se involucran aquellos derechos que tienen contenido social tales como salud, alimentación, educación, vivienda, asistencia, empleo. Asimismo, otros derechos humanos más ligados a su concepción clásica- son los que posibilitan las condiciones materiales y simbólicas necesarias para el ejercicio de aquellos. Aquí podemos mencionar la igualdad y no discriminación, la libertad de expresión, la participación política, el acceso a la justicia, entre otros de corte similar (Abramovich, 2004; 2006).

A partir de la asociación entre políticas sociales y derechos se señalan estándares interpretativos -derivados de organismos de derechos humanos- aplicables a las primeras: el contenido mínimo de los derechos y la universalidad; la utilización del máximo de los recursos disponibles; la progresividad y no regresividad; la igualdad y no discriminación; el acceso a la 
justicia y los mecanismos de reclamo; la producción y el acceso a la información; la participación en el diseño de las políticas (Pautassi, 2010).

Tal como se señaló al comienzo, en los últimos años -motivados por el particular contexto sociohistórico que inició en el nuevo siglo- ha crecido la producción científica que indaga políticas sociales desde el enfoque de derechos. El primer antecedente se ubica en la declaración de los Objetivos del Desarrollo del Milenio de la Organización de Naciones Unidas (ODM) los cuales debían cumplirse en 2015. A partir de ello -a nivel nacional e internacional- los estudios sobre derechos y políticas sociales han ido incrementando (Pautassi, 2004; Guendel, 2007; Oyarzún, Dávila, Hativobic, Ghiardo, 2008; Mazzola, 2012; Llobet, 2013; Pautassi, et. al., 2013; Arcidiácono, 2015; Cristini, 2016; Becher, 2017).

\section{Los efectos subjetivos}

A partir de los aportes del posestructuralismo en las teorías de la subjetividad, en donde podemos ubicar a Guattari (1986) y a Deleuze (1995), comienza a adquirir significancia la presencia de la otredad como un aspecto que incide en la construcción de las subjetividades. Por ello las redes de sociabilidad empiezan a ser consideradas un elemento de análisis relevante en los estudios sobre inclusión-exclusión desde fines de la década de 1980.

Ya sea que se utilicen nociones tales como descalificación (Paugam, 1991) desafiliación (Castel, 2004) o desligadura (Autés, 2004) todos estos autores -ya sea desde perspectivas teóricas o empíricas- coinciden en que la exclusión daña lazos sociales o redes de sociabilidad y del mismo modo produce procesos de desubjetivación (De Gaulejac y Taboada-Léonetti, 1994). Michel Autés (2004) en su texto Tres formas de desligadura en el que revisa los enfoques teóricos propuestos por los autores antes citados afirma: "La exclusión es un nombre puesto a los infortunios de la época, que dañan las articulaciones entre las diferentes esferas de la vida social, entre los diferentes universos, entre los diferentes "mundos", como se dice ahora" (p. 17). Continúa

Cuando hablamos de exclusión, estamos ante algo que implica ruptura del lazo. Detrás de esta conceptualización se cuestiona la propia cohesión social: ¿qué cosa asegura la cohesión, y cómo devolver un sentido a la pertenencia de los individuos tanto como a su identidad? ¿Cómo hacer algo del orden de lo social pero esta vez tomado como la "sociedad"? (p. 18).

Autores anglosajones y británicos también coinciden en la relación entre la exclusión y las redes de sociabilidad. Peter Townsend (1979) señala que una comprensión adecuada de la pobreza no debe restringirse a la preocupación por la satisfacción de las denominadas 
necesidades elementales sino que debe incorporar la incapacidad de las personas para integrarse en la vida habitual de la sociedad. Por su parte Burchardt et. al. (2002) afirma:

... un individuo está excluido socialmente si no forma parte de actividades clave de la sociedad en la cual vive... el individuo no participa de las mismas por razones más allá de su control (en referencia a la exclusión) pero le gustaría participar (pp. 30-31. Traducción propia) (el agregado entre paréntesis nos corresponde).

Si la exclusión afecta la cohesión social y las redes de sociabilidad, por consiguiente a la inclusión y los derechos les corresponde lo contrario: reconstruir lazos sociales y reparar tales redes. En Argentina es la investigadora Valeria Llobet (2013) quien ha insistido en esta cuestión y en la posibilidad de las políticas sociales de constituirse en reservorios de lazos afectivos subjetivos y restaurar las huellas dejadas por las diferentes modalidades de la exclusión:

Las transformaciones (en referencia a los efectos de las políticas sociales que incorpora en su estudio) tendieron a concentrarse más y más en el escenario de la subjetividad, y los éxitos, a concebirse en el plano vincular, consolidando una imagen de la intervención enfocada en la instalación de los programas como reservorios afectivos y de lazo social (p. 13) (el agregado entre paréntesis nos corresponde).

Por otra parte, la exploración de las subjetividades en el marco de la inclusión y los derechos incorpora la importancia de lo social por cuanto el contexto es un elemento fundamental que aporta a su construcción y a este aspecto -que permea todas las dimensiones de nuestra investigación- lo incorporamos particularmente en una de las variables que mencionamos más adelante. Dice Autés (2004):

¿Qué es lo social? No algo que tenga una existencia en sí: lo social es ante todo una articulación de la esfera económica con la esfera política... se anexa a estas dos esferas otros dos importantes campos de la actividad humana: el cultural y el doméstico. Todo esto va a combinarse para formar lo social (pp. 16-17).

De allí que las posibilidades de inclusión y derechos no pueden ser consideradas un fenómeno unidimensional pues involucran los diferentes ámbitos de la actividad humana. Dice Kessler (2016): "Diferentes indicadores han integrado otras dimensiones como salud, educación, vivienda, a las que se han incorporado condiciones del medio ambiente, acceso a la justicia, respeto o reconocimiento de la diversidad, entre otras" (p. 29).

La última área que se ha incorporado de manera particular al ámbito de los procesos de inclusión es la de los consumos culturales. Si bien -tal como señala Grassi (2008)- mucho se ha insistido en la distinción entre unas necesidades básicas y otras no tanto, la mayoría de la sociedad satisface tales consumos sin mucho prurito. Ello nos muestra que las necesidades sociales son principalmente una construcción condicionada por los diferentes tiempos históricos. Tanto Heller (1986) como Fraser (1991) destacan la intervención del Estado en la 
definición de las necesidades y en ello el modo en que se deja de lado su carácter eminentemente político y colectivo.

Bauman (2014) es quien se ha dedicado con exhaustividad al estudio de los consumos culturales en la contemporaneidad. El sociólogo destaca el modo en que tales consumos construyen subjetividades en términos de ciudadanos a quienes se los denomina de pleno derecho y otros a quienes se los califica como fracasados. Por ello la importancia de que las políticas sociales vayan incorporando tales aspectos. Estudios sociales mencionan el impacto positivo del acceso a consumos culturales en el programa social Asignación Universal por Hijo para Protección Social implementado en Argentina en el año 2009 y con vigencia en la actualidad (Mazzola, 2012; Pautassi et. al., 2013; Becher, 2017).

Tal como señala Karsz (2004) -y en la Argentina Lo Vuolo et. al. (2004)- nadie puede estar en su totalidad excluido de las distintas esferas de la vida social y al mismo tiempo solo es posible estarlo respecto de determinados ámbitos, lo cual se vincula con la multidimensionaldad propia de tales procesos: "Si se busca bien, uno está siempre excluido con relación a alguien, uno está siempre excluido de algo" (p. 141). Si nadie puede estar excluido en su totalidad de la sociedad, del mismo modo nadie puede estarlo en referencia a la inclusión y el acceso a derechos. Ello pone en evidencia las limitaciones propias de tales procesos pues su diversidad posibilita particulares modos de estar incluido y respecto de determinados espacios y derechos. No es posible experimentarlo en su totalidad.

\section{LAS BRECHAS DE GÉNERO Y EL DESAFÍO PARA LA INCLUSIÓN}

Si bien la distinción entre sexo y género ocupa vastos espacios dentro de los anaqueles de las investigaciones sociales, nunca es innecesario recordar su distinción pues en ello se basa el concepto de brechas de género (Kabeer, 1991; 1999). Tanto uno como otro son construcciones sociales, pero con género expresamos el conjunto de características y comportamientos como así también roles, funciones y valoraciones impuestas dicotómicamente a cada sexo a través de procesos de socialización que son mantenidos y reforzados por la ideología e instituciones patriarcales (Facio y Fries, [1999] 2005).

Tales representaciones dicotómicas construyen diferencias de género que operan como condiciones que estructuran las distintas esferas de la actividad humana y de allí el desafío para la inclusión social y el acceso a derechos. Pautassi (2007) señala: 
Resulta necesario garantizar la llamada igualdad material o estructural. La misma parte del reconocimiento de que ciertos sectores de la población no gozan de todos los valores y medios para desarrollarse como personas y para participar en pleno en la organización económica, política y social; esto requiere de la adopción de medidas especiales de equiparación ( $p$. 27).

En este caso tales procesos no solo se enfrentan a una posible situación de exclusión sino que se agrega otra de discriminación estructural reproducida históricamente. Por ello apostar por una justicia social en donde la autonomía de las mujeres sea prioridad constituye un importante estímulo para superar esas desigualdades. François Dubet (2011) menciona que en la actualidad existen dos grandes concepciones de justicia social y ambas pendulan en torno a la distinción entre igualdad de oportunidades y de posiciones. Aunque ambas coinciden por cuanto buscan reducir el desequilibrio que existe en las sociedades democráticas entre la afirmación de la igualdad de todos los ciudadanos y las inequidades sociales existentes en la realidad social. Allí radica -tal como señalaba Pautassi en su cita anterior- la distinción entre la igualdad formal y la material.

Si bien ambas concepciones son similares en su objetivo, lo son totalmente dispares en cuanto al modo que proponen para lograrlo. En la igualdad de oportunidades lo que prima es la posibilidad de ofrecer a todos los ciudadanos -por utópico que parezca- la oportunidad de ocupar iguales posiciones en donde la meritocracia es la que define el ascenso o no a tal posición. Por el contrario, la igualdad de posiciones busca acercar espacios entre las disparidades que existen en los principios que organizan la estructura social.

La igualdad de oportunidades se garantiza asegurando a todas las mujeres la posibilidad de acceder al derecho al trabajo en iguales condiciones que los varones, en cambio, la igualdad de posiciones buscará reducir las brechas salariales entre unos y otros. La igualdad de oportunidades permite a las mujeres acceder a una asignación universal por hijo, en cambio, la igualdad de posiciones reduciría las cargas de cuidado que imponen tales programas sobre las mismas. La igualdad de oportunidades garantiza a las mujeres el ingreso a cargos legislativos mediante el establecimiento de un cupo femenino, en cambio, la igualdad de posiciones busca su incidencia real en la toma de decisiones.

Sin dudas, tanto una como otra concepción son pasibles de críticas y sin dudas el discurso de las oportunidades tiene importantes efectos performativos -pues es sumamente atractivo- en lo relativo a los derechos humanos y a la reducción de las desigualdades de género, pero -tal como señala el sociólogo francés- el cambio real se producirá cuando 
logremos modificar las estructuras o posiciones entre los géneros y de ese modo contribuir a una igualdad, quizá menos atractiva en términos político-partidarios y mediáticos, más efectiva.

En términos de desigualdades de género, para lograr la igualdad de oportunidades entre varones y mujeres primero es necesario bregar por la igualdad de posiciones, pues de lo contrario quedamos al arbitrio de un principio meritocrático que por medio de regulaciones normativo-androcéntricas beneficia al colectivo masculino. Por ello las políticas sociales -como principales mecanismos contemporáneos que se constituyen en la región para lograr el objetivo de inclusión social- deben apostar a la universalización como criterio para su definición.

\section{FINALIZAR PARA INICIAR: POSIBLES DIMENSIONES DE ANÁLISIS Y EXPERIENCIAS DE INVESTIGACIÓN SOCIAL}

Poner en práctica una propuesta teórica involucra otra de corte metodológico pues -tal como señala Guber (2010)- es allí donde se ponen a andar, dando inicio a la investigación, los presupuestos teóricos antes explicitados. En tal sentido, vale la pena proponer algunas dimensiones de análisis, o variables conceptuales, respecto de las cuales los lectores tienen la licencia de modificarlas o bien de proponer unas distintas o simplemente desecharlas. Tales dimensiones surgen de nuestra experiencia en los estudios sociales sobre juventudes y su clivaje con género en el marco de políticas sociales como parte de las investigaciones antes mencionadas.

En la construcción de las variables hemos tenido en cuenta los aportes de Delor y Hubert (2000) en su texto Revisiting the concept of "vulnerability" en donde proponen dimensiones de análisis en referencia a lo que denominan subjetividades vulnerabilizadas. Castel (2004) es quien distingue entre tres zonas de la vida social. La primera es la integración y refleja la realidad de aquellos que tienen un trabajo regular y vastos soportes de sociabilidad. Por su parte, la zona de vulnerabilidad refiere a circunstancias tales como la del trabajo precario y la de situaciones relacionales inestables. Y la última zona corresponde a la de la exclusión donde pueden caer tanto los integrados como los vulnerables y las características de cada una de tales zonas -condición de empleabilidad y sociabilidad- se presentan como carencia en extremo.

Si bien hemos considerado la propuesta de los autores, también la hemos adaptado a las características contextuales de nuestro país y de las categorías teóricas en las cuales desarrollamos nuestras experiencias de investigación. A partir de ello proponemos tres 
variables: la institucionalidad social, las trayectorias personales y el contexto situacional. Todas ellas presentan particularidades pero, al mismo tiempo, todas hacen posible la exploración de las subjetividades -incluida la instancia de responsabilidad estatal- en juventudes y su intersección con género.

En la primera dimensión, institucionalidad social, se observan particularmente las instituciones y organizaciones involucradas en la implementación del programa social de que se trate y la medida en que contribuyen a construir espacios de inclusión y derechos. Es Repetto (2010) quien define dicha institucionalidad como el conjunto de organizaciones involucradas en la instancia de ejecución de la política social y por consiguiente ligadas a la burocracia estatal. De allí que será posible bucear en las dinámicas propias de cada programación pública y el modo en que las juventudes se fueron aproximando a las mismas.

Solo cuando incorporamos la dimensión de trayectorias personales podemos indagar con mayor profundidad la dimensión biográfica de los procesos de inclusión social y acceso a derechos, como así también las que contribuyen a la reproducción social. Por ello en esta instancia la categoría teórica vida cotidiana adquiere una particular significación por cuanto refiere al conjunto de actividades que realizan los sujetos particulares y contribuyen a la reproducción social (Heller, 1987). Al mismo tiempo, se constituye en un espacio propicio para indagar el modo en que se construyen las subjetividades con especial referencia al significado que se le atribuye a la presencia del otro en el marco de las relaciones intersubjetivas (Castro, 2012). En esta dimensión se podrá indagar el impacto de los procesos de inclusión y derechos en las actividades que las juventudes desarrollan en su vida cotidiana y el espacio que le otorgan a cada una de ellas.

Cook y Cusack (2010) definen al contexto situacional como el conjunto de condiciones predispuestas. Por ello se presenta como un escenario propicio para analizar el vínculo entre las condiciones propias de las modalidades que asumen las juventudes en su intersección con género en relación con otros clivajes identitarios. A partir de ello, se constituye en un ámbito oportuno para explorar el significado que adquieren las relaciones intersubjetivas en el contexto específico en que se desarrollan y de allí que tal especificidad puede conducir a incluir las particularidades del escenario sociopolítico en que se producen.

Las experiencias de investigación a las que haremos referencia se enmarcan en una tesis doctoral en curso que tiene como objetivo explorar el modo en que se construyen las subjetividades de los destinatarios jóvenes de dos programas sociales -uno provincial y otro del nivel nacional cuyos objetivos son la inclusión social y el acceso a derechos- a partir de conocer 
sus experiencias intersubjetivas y las significaciones que derivan de ellas. El programa provincial se denomina Plan de Inclusión Social Trabajo por San Luis (PISTS) y el nacional es el Programa de Respaldo a Estudiantes Argentinos (PROGRESAR). El primero de ellos comenzó a implementarse en el año 2003 y como una medida social que tenía principalmente la intención de contribuir a paliar las consecuencias sociales de la crisis argentina de aquel periodo. Por su parte, el PROGRESAR inició su recorrido casi doce años después -a comienzos de 2014- en donde el escenario sociopolítico era disímil del anterior por cuanto al menos parcialmente se habían superado algunos aspectos de la crisis de principios de siglo. Los dos programas sociales son transferencias condicionadas que tienen como objetivo la capacitación laboral y futura inserción en el empleo -en el caso del PISTS- y la continuación o finalización de los estudios formales de primer nivel o universitario en el Programa de Respaldo a Estudiantes Argentinos. Vale señalar que como consecuencia de las exclusiones las juventudes destinatarias de los programas presentan como particularidad dominante escasos soportes de sociabilidad ligados a contextos inclusivos.

Al mismo tiempo, esta investigación se desarrolla en una provincia del interior del país la de San Luis- y ello agrega una particularidad interesante por cuanto son escasos los estudios tanto en ciencias sociales como en juventudes y género que se realizan en tales provincias. Sumado a ello, las particularidades materiales y simbólicas que son propias de la construcción territorial y que pudimos advertir especialmente en la variable sobre contexto situacional.

Tal como hemos señalado, en función de los aportes de diferentes autores, las redes de sociabilidad son un aspecto clave en nuestro enfoque teórico-metodológico sobre la producción subjetiva enmarcada en la inclusión y el acceso a derechos. En ese sentido vamos a señalar algunos aspectos centrales de dicha dimensión en las diferentes variables y respecto de la unidad de análisis que hemos construido para nuestra investigación. El modo en que las juventudes se van aproximando a la burocracia estatal -ya sea para acceder a las prestaciones del programa o cuando ya están insertos para cumplir con sus condiciones- se encuentra vinculado con las redes de sociabilidad previamente construidas y con los soportes que ya poseen en el marco de tales burocracias. De allí que es muy frecuente que los jóvenes conozcan los programas sociales debido a que algún familiar o amigo trabaja en la administración pública o sus padres o amigos han formado parte de tales programas. Las redes de sociabilidad que poseen los jóvenes están vinculadas a sus experiencias previas con el empleo y si bien lo que predomina es la precarización tanto entre varones como mujeres, son estas últimas las que 
poseen menores redes dada su experiencia de empleo principalmente en el ámbito privado, y ligada a las tareas de cuidado, que en la esfera pública.

En lo referido a las trayectorias personales, pudimos advertir que las posibilidades de construcción de nuevas redes de sociabilidad -que efectivamente se dan en el marco de los programas- nuevamente están condicionadas por el modo en que se construyen las relaciones sociales de género en la cotidianidad juvenil. Ello por cuanto no todas pero la mayoría de las nuevas redes que construyen las mujeres están ligadas con las tareas de cuidado que realizan como madres o como cuidadoras de otros familiares o de ajenos. A partir de tal circunstancia, sus procesos inclusivos giran en torno a esas experiencias -en donde la reproducción de estereotipos encuentra terreno fértil- y las mujeres se preocupan más por la inclusión y el acceso a derechos de sus hijos que la propia. Distinto es lo que sucede en el caso de los varones en donde las redes de sociabilidad que proporcionan los programas les ha facilitado la inserción, precaria en algunos casos y en otros no, en diversos empleos ligados -también en algunos casos- incluso con sus propias preferencias laborales. Del mismo modo esta situación se reproduce en lo referido a la educación por cuanto son los varones -y dado que no llevan a cabo tareas de cuidado- quienes han podido no solo completar sino hacerlo con mejor rendimiento académico sus trayectorias educativas formales.

Si bien la variable de contexto situacional nos ha proporcionado datos tanto respecto de la primera como de la segunda dimensión, nos vamos a centrar en lo referido a la influencia del escenario sociopolítico sanluiseño en los procesos inclusivos y de derechos de las juventudes. Dicho escenario se caracteriza por la presencia de un mismo proyecto político representado por los hermanos Rodríguez Saá -quienes pertenecen al Partido Justicialista- presente desde el año 1983 hasta la actualidad. Ello implica que las subjetividades juveniles con las cuales hemos trabajado en nuestra investigación son una generación que ha transcurrido toda su existencia bajo la persistencia de un mismo estilo político, de allí que algunos aspectos hegemónicos -en términos gramscianos- han sido naturalizados culturalmente por las juventudes. Si bien las relaciones de poder y de significación -tal como plantea Foucault (1988)- que se construyen en el marco de un escenario sociopolítico de tales características, y si fuera distinto también, se enfrenta a modalidades subjetivas de resistencia más o menos explícitas, tales modalidades son distintas entre varones y mujeres. Los primeros cuentan con soportes de sociabilidad y vínculos con la burocracia estatal más desarrollados -dadas las condiciones que comentábamos anteriormente- lo cual les permite sortear las consecuencias que derivan de esas resistencias. Por el contrario, las mujeres no cuentan con tales redes y ello las convierte en subjetividades 
más proclives a construir una relación de poder y de significación-que es asimétrica al igual que la de género- respecto de quienes detentan ese poder en donde las resistencias son menores.

De tales experiencias de investigación podemos advertir que la juventud en su intersección con género -en tanto dimensiones constitutivas de las subjetividades- son transversales a la totalidad de los aspectos que comprenden los procesos de inclusión y acceso a derechos que experimentan. Sin embargo, también podemos advertir que si bien juventudes y género estructuran la mayoría de los aspectos que comprenden sus procesos inclusivos, del mismo modo se encuentran condicionados por otros factores. Entre ellos los más recurrentes son la condición de empleo, la familiar, la política y la educativa. A partir de los resultados obtenidos en el marco de las indagaciones mencionadas, es posible señalar que cada una de esas condiciones se presenta con características particulares y de ese modo influyen en la inclusión y el acceso a derechos y en ello en las redes de sociabilidad construidas. Tanto entre jóvenes varones como mujeres lo que prima es la precarización, en referencia a la condición de empleo; el desconocimiento de la importancia de las tareas reproductivas que llevan a cabo las mujeres aportando de ese modo a una particular constitución de la condición familiar; un relativo grado de participación social pero no menos significativo en algunas historias de vida; y escasos niveles de instrucción en algunos casos o trayectorias educativas truncas en otros más numerosos.

A modo de hipótesis general de los resultados preliminares, podemos advertir que los espacios de socialización propuestos por los programas han reconstruido lazos sociales por cuanto se han constituido en espacios en donde la subjetividad de sus destinatarios previamente vulnerabilizada por la agudización de las exclusiones- reposa frente al temor de la incertidumbre del mundo social para los jóvenes varones y mujeres actuales y logra reponerse para enfrentarlo con nuevas herramientas. Dichas herramientas son producto principalmente de nuevos soportes de sociabilidad con los cuales los miembros del colectivo socio-generacional se preparan para enfrentar las diversas vicisitudes de la vida social. Ello muestra que los espacios instituidos por los programas se constituyen principalmente en reservorios de lazos sociales y no tanto en instancias de formación respecto del empleo o la educación, de acuerdo con los objetivos de los programas. 


\section{CONCLUSIÓN}

No es sencillo intentar teorizar en el marco de una ciencia social en donde principalmente han predominado y han sido particularmente valorados los estudios de corte empírico. Esto mismo decía Luhmann ([1984] 1998) a fines del siglo XX y respecto de su teoría de los sistemas sociales. Ya han transcurrido varios años pero tal afirmación continúa vigente. Ello sucede por cuanto exponer una propuesta teórica coloca a los investigadores frente a cierta vulnerabilidad dado que se someten a la consideración de otros pares que pueden considerar apropiados o no sus planteos. Aunque no es menos cierto que los enfoques metodológicos, tanto cualitativos como cuantitativos, constituyen un importante estímulo para animarse a teorizar por cuanto la construcción interactiva entre el marco de referencia y el campo empírico supone respecto de esa teoría un importante respaldo.

Animarse a teorizar puede no ser sencillo pero no es menos cierto que toda investigación social tiene como principal objetivo al momento de su finalización construir nueva teoría o incidir en una ya existente. Allí radica la justificación de lo que metodológicamente se denomina la construcción del dato cualitativo. Por ello, el principal objetivo de este texto es proponer una mirada teórica sobre la inclusión social y el acceso a derechos en juventudes en su intersección con género, en donde lo que predomina es una mirada socio-antropológica. Ello supone otro desafío: tanto inclusión social como acceso a derechos se han convertido en dos significantes recurrentes por parte del discurso político y mediático y de allí su influencia en el social. Al mismo tiempo, esa circunstancia coloca a las ciencias sociales y sus investigadores frente a la necesidad que siempre los ha interpelado: aportar desde determinados estándares epistemológicos y metodológicos a la construcción de categorías teóricas. Seguramente y no es una cuestión menor, esta sea una de las principales modalidades por medio de las cuales los científicos sociales podamos incidir en otras comunidades distintas de las nuestras.

Proponer una mirada socio-antropológica sobre la inclusión y el acceso a derechos respecto de las juventudes y género posibilita de manera particular bucear en el modo en que se construyen y se significan los vínculos, en el marco de un contexto sociocultural, con el otro, ya sea el Estado o los otros destinatarios o no destinatarios de los programas sociales. Sin dudas toda mirada teórica involucra otra metodológica y en ese sentido hemos propuesto tres variables conceptuales de análisis de procesos inclusivos y de derechos. Tales variables son producto y han sido útiles respecto de nuestras investigaciones, pero ello no excluye la 
posibilidad de proponer otras distintas o de solo considerar algunos aspectos de éstas pues en definitiva las mismas son producto de influencias teóricas y metodológicas previas.

Si bien hemos expuesto brevemente algunos resultados obtenidos en el marco de nuestras experiencias de investigación los mismos tienen dos propósitos: por un lado, dar cuenta de esa construcción interactiva entre el marco de referencia y los datos empíricos a los cuales referimos previamente, y por otro, ser un estímulo para llevar a cabo investigaciones sociales desde la perspectiva teórico-metodológica que planteamos en el artículo.

Finalmente, y creemos que este es nuestro principal aporte y dato cualitativo tal como señalábamos previamente, la diversidad de los escenarios inclusivos y de derechos muestran su constante limitación, pues del mismo modo que la exclusión nunca es total, la inclusión tampoco puede serlo. En ese sentido, proponemos como nuevo significante para nominar tales procesos la propuesta de pluralizar el término y hablar de inclusiones y no de inclusión, y mucho menos de inclusión social pues como hemos visto lo social es representativo de la totalidad de las esferas de la vida. Asimismo, y tal como ha sucedido con otras categorías teóricas, esto también muestra que las inclusiones se encuentran en permanente construcción y por consiguiente no son estáticas: hoy se puede estar incluido respecto de determinadas esferas de la vida social, pero mañana no se sabe.

\section{BIBLIOGRAFÍA CONSULTADA}

ABRAMOVICH, V. (2004). Una aproximación al enfoque de derechos en las estrategias y políticas de desarrollo de América Latina. Centro de Estudios Legales y Sociales (CELS). Documento preparado para "Derechos y desarrollo en América Latina: una reunión de trabajo". Chile. (2006). Una aproximación al enfoque de derechos en las estrategias y políticas de desarrollo. Revista de la CEPAL, 88, 3550.

ANDRENACCI, L. (2010). Política social y ciudadanía en América Latina contemporánea: una contribución al debate acerca de las transformaciones de política social de la región en la primera década del Siglo XXI. Ponencia presentada en el II Congreso Latinoamericano y Caribeño de Ciencias Sociales. México.

ARCIDIÁCONO, P. (2015). Diez años de transferencias a las familias con hijos en la Argentina (20032013): una propuesta de periodización. En L. Pautassi y G. Gamallo (Comps.), El bienestar en brechas: las políticas sociales en la Argentina de la posconvertibilidad (pp. 7390). Buenos Aires: Biblos.

AUTÉS, M. (2004). Tres formas de desligadura. En S. Karsz (Coord.), La exclusión: bordeando sus fronteras. Definiciones y matices (pp. 1553). Barcelona: Editorial Gedisa. 
BAUMAN, Z. (2014). ¿La riqueza de unos pocos nos beneficia a todos? Ciudad de Buenos Aires: Paidós. Traducido por Alicia Capel Tatjer.

BECHER, Y. (2017). Cartografías socioestatales y subjetividades. Un recorrido sobre programas sociales en la contemporaneidad. Buenos Aires: Teseo.

BIERNAT, C. y Ramacciotti, K. (2012). Preguntas y herramientas para el análisis de las políticas sociales. En C. Biernat y K. Ramacciotti (Ed.), Políticas sociales. Entre demandas y resistencias. Argentina 19301970 (pp. 936). Buenos Aires: Biblos.

BURCHARDT, T., Le Grand, T. y Piachaud, D. (2002). Degrees of exclusion: developing a dynamic, multidimensional measure. In J. Hills, J. Le Grand and D. Piachaud (Eds.), Understanding social exclusion (pp. 3043). Oxford: Oxford University Press.

CASTEL, R. (2004). Encuadre de la exclusión. En S. Karsz (Coord.), La exclusión: bordeando sus fronteras. Definiciones y matices (pp. 5586). Barcelona: Editorial Gedisa.

CASTRO, G. (2012). Los jóvenes y la vida cotidiana. Construcción de la subjetividad y la identidad social en sociedades con cambios socioculturales. Tesis de doctorado (inédita). Universidad Nacional de San Luis.

COMITÉ PARA LA ELIMINACIÓN DE LA DISCRIMINACIÓN CONTRA LA MUJER (2010). Observaciones finales para Argentina. Naciones Unidas.

COOK, R. J. y Cusack, S. (2010). Estereotipos de género. Perspectivas legales transnacionales. Profamilia. Traducido por Andrea Parra.

CRISTINI, R. (2016). El derecho de participación de niños, niñas y adolescentes (NNA) en la escuela: acción política y procesos instituyentes. Un estudio de casos en escuelas preuniversitarias de la Ciudad de Córdoba. Tesis de doctorado (inédita). Universidad Nacional de Córdoba.

DAS, V. y Pool, D. ([2004] 2008). El Estado y sus márgenes. Etnografías comparadas. Cuadernos de Antropología Social, 27, 1952. Universidad de Buenos Aires.

DE GAULEJAC V. y TaboadaLéonetti, I. (1994). La Lutte des places. París: Desclée de Brouwer.

DELEUZE, G. (1995). Conversaciones 19721990. Valencia: Pre Textos.

DELOR, F. y HUBERT, M. (2000). Revisiting the concept of vulnerability. Social science and medicine, 50, 15571570.

DUBET, F. (2011). Repensar la justicia social. Contra el mito de la igualdad de oportunidades. Buenos Aires: Siglo XXI Editores.

ESPINGANDERSEN, G. (1990). The three worlds of welfare capitalism. Cambridge: Polity Press.

FACIO, A. y FRIES, L. (1999 [2005]). Feminismo, género y patriarcado. Revista sobre Enseñanza del Derecho de Buenos Aires, 3 (6), 259294. 
FRASER, N. (1991). La lucha por las necesidades: esbozo de una teoría crítica socialista feminista de la cultura política del capitalismo tardío. Revista Debate Feminista, 2 (3).

FOUCAULT, M. (1988). El sujeto y el poder. Revista Mexicana de Sociología, 50 (3), 320.

GAMALLO, G. (2015). Aproximación al concepto de brecha de bienestar. En L. Pautassi y G. Gamallo (Comps.), El bienestar en brechas: las políticas sociales en la Argentina de la posconvertibilidad (pp. 3148). Buenos Aires: Biblos.

GRASSI, E. (2008). La política social, las necesidades sociales y el principio de la igualdad: reflexiones para un debate postneoliberal. En J. P. Jarrín, Es posible pensar una nueva política social para América Latina (pp. 2968). Ecuador: FLACSO.

GUATTARI, F. (1986 [2006]). Subjetividad e historia. En Guattari, F. y Rolnik, S., Micropolítica. Cartografías del deseo (39147). Madrid: Traficantes de Sueños.

GUENDEL, L. (2007). La encrucijada del enfoque de derechos: pensando y haciendo la política pública de otra manera. Ponencia presentada al VIII Seminario de formación en DESC "Una mirada a las políticas públicas desde los derechos humanos".

GUBER, R. (2010). El proyecto de investigación en ciencias sociales: acepción, concepción y redacción. En Construcción de proyectos en Ciencias Sociales: investigación cualitativa, acción social y gestión cultural. CAICYTCONICET.

HELLER, A. (1986). Teoría de las necesidades en Marx. Barcelona: Península. Traducción de J. Yvars.

(1987). Sociología de la vida cotidiana. Barcelona: Ediciones Península.

KABEER, N. (1991). Gender, development, and training: Raising awarness in the planning process. Development in practice, 1 (3), 185195.

(1999). Resources, Agency, Achievements: Reflections on the Measurment of Women's Empowerment. Development and change, 30, 435464.

KARSZ, S. (2004). La exclusión: concepto falso, problema verdadero. En S. Karsz (Coord.), La exclusión: bordeando sus fronteras. Definiciones y matices (pp. 133214). Barcelona: Editorial Gedisa.

KESSLER, G. (2016). Controversias sobre la desigualdad: Argentina 20032013. Buenos Aires: Fondo de Cultura Económica.

LO VUOLO, R., Barbeito, A., Pautassi, L. y Rodríguez, C. (2004). La pobreza... de la política contra la pobreza. Buenos Aires: Miño y DávilaCiepp.

LUHMANN, N. ([1984] 1998). Sistemas sociales: lineamientos para una teoría general. Barcelona: Anthropos. México: Universidad Iberoamericana. Colombia: CEJAPontificia Universidad Javeriana.

Llobet, V. (2013) (Comp.). Sentidos de la exclusión social. Beneficiarios, necesidades y prácticas en políticas sociales para la inclusión de niños y jóvenes. Buenos Aires: Biblos. 
MAZZOLA, R. (2012). Nuevo paradigma: la Asignación Universal por Hijo en Argentina. Buenos Aires: Prometeo Libros.

NÚÑEZ, P., VÁZQUEZ, M. y Vommaro, P. (2015). Entre la inclusión y la participación. Una revisión de las políticas públicas de juventud en la Argentina actual. En H. Cubides C., S. Borelli, R. Unda, M. Vázquez (Edrs.), Juventudes Latinoamericanas. Prácticas socioculturales, políticas y políticas públicas. Buenos Aires: CLACSO.

ORGANIZACIÓN DE NACIONES UNIDAS (ONU). Oficina del Alto Comisionado de las Naciones Unidas para los Derechos Humanos (OACDH) (2006). Preguntas frecuentes sobre el enfoque de los derechos humanos en la cooperación para el desarrollo. New York y Ginebra: Naciones Unidas.

OYARZÚN, A., DÁVILA, O., Ghiardo, F., Hatibovic, F. (2008). ¿Enfoque de derechos o enfoque de necesidades? Chile: SENAME, Ediciones CIDPA.

PAUGAM, S. (1991). La Disqualification sociale. Essai sur la nouvelle pauvreté. París: Puf.

PAUTASSI, L. (2004). Beneficios y beneficiarias: análisis del Programa Jefes y Jefas de Hogar Desocupados en Argentina. En M. E. Valenzuela (Ed.), Políticas de empleo para superar la pobreza: Argentina. Santiago de Chile: OIT.

(2007). ¡Cuánto trabajo mujer! El género y las relaciones laborales. Buenos Aires: Capital Intelectual.

(2010). Indicadores en materia de derechos económicos, sociales y culturales. Más allá de la medición. En V. Abromovich y L. Pautassi (Comps.), La medición de derechos en las políticas sociales (pp. 187). Buenos Aires: Del Puerto.

(2015). Introducción: situando el bienestar, identificando brechas. En L. Pautassi y G. Gamallo (Comps.), El bienestar en brechas: las políticas sociales en la Argentina de la posconvertibilidad (pp. 1529). Buenos Aires: Biblos.

PAUTASSI, L., ARCIDIÁCONO, P. y Straschnoy, M. (2013). Asignación Universal por Hijo para la Protección Social de la Argentina. Entre la satisfacción de necesidades y el reconocimiento de derechos. Santiago de Chile: Naciones UnidasCEPAL.

REPETTO, F. (2010). Protección social en América Latina: la búsqueda de una integralidad con enfoque de derechos. Revista del CLAD Reforma y Democracia, 47, Caracas.

RODRÍGUEZ ENRÍQUEZ, C. (2005). Economía feminista y economía de cuidado. Aportes conceptuales para el estudio de la desigualdad. Nueva Sociedad, 256, 3044.

SALVIA, A. y Tuñón, I. (2006). Los jóvenes y el mundo del trabajo en la Argentina actual: joven argentino. Encrucijadas, 36. Universidad de Buenos Aires.

(2007). Jóvenes excluidos: Límites y alcances de las políticas públicas de inclusión social a través de la capacitación laboral. Revista de Estudios Regionales y Mercado de Trabajo, 3, 5168. Consultada el 12 de Julio de 2015, http://www.memoria.fahce.unlp.edu.ar/art_revistas/pr.4336/pr.4336

SEN, A. (1997). Capital humano y capacidad humana. Obtenida el 15 de septiembre de 2004, de www.redvertice.com/fep. 
(2000). Desarrollo y libertad. Bogotá: Editorial Planeta.

(2003). ¿Qué impacto puede tener la ética? Trabajo presentado en la Reunión Internacional sobre "Ética y Desarrollo" del Banco Interamericano de Desarrollo en colaboración con el Gobierno de Noruega.

TONON, G. (2008). Estudiar las desigualdades sociales en Argentina: el desafío de construir nuevos indicadores desde la mirada de las capabilities. En G. Tonon (Coord.), Desigualdades sociales y oportunidades ciudadanas. Buenos Aires: Espacio editorial.

TOWNSEND, P. (1979). Poverty in the United Kingdom. Harmondsworth: Penguin.

Trabalho enviado em 12 de janeiro de 2018.

Aceito em 25 de março de 2018. 